\title{
Integrated light and electron microscopy continuum resolution imaging of 3D cell cultures
}

\section{Edoardo D’Imprima ${ }^{\S a}$, Marta Garcia Montero ${ }^{\S b c}$, Sylwia Gawrzak ${ }^{\S b d}$, Paolo Ronchi e, levgeniia Zagoriy ${ }^{a}$, Yannick Schwab ${ }^{\text {be }}$, Martin Jechlinger ${ }^{\text {bf }}$ and Julia Mahamid ${ }^{\text {ag }}$}

a

Structural and Computational Biology Unit, European Molecular Biology Laboratory (EMBL), Meyerhofstrasse 1, 69117 Heidelberg, Germany

Cell Biology and Biophysics Unit, European Molecular Biology Laboratory, Meyerhofstrasse 1, 69117 Heidelberg, Germany

C Current address: VitroScan, Leiden Bio Science Park, Biopartner 2, J.H Oortweg $212333 \mathrm{CH}$ Leiden, The Netherlands

d

Current address: Functional Genomics, Cellzome GmbH - a GlaxoSmithKlein company, Meyerhofstrasse 1, 69117 Heidelberg, Germany

e Electron Microscopy Core Facility, European Molecular Biology Laboratory, Meyerhofstrasse 1, 69117 Heidelberg, Germany

Current address: MOLIT Institut gGmbH, Im Zukunftspark 10, 74076 Heilbronn, Germany

9 Lead contact.

Correspondence should be addressed to: martin.jechlinger@embl.de, julia.mahamid@embl.de $\S_{\text {Equal contribution }}$

\section{Summary}

3D cell cultures, in particular organoids, recapitulate tissue architectures and are emerging model systems to investigate healthy or diseased tissues and organs. Understanding the complex cellular sociology of organoid requires the integration of diverse imaging modalities covering different spatial and temporal resolutions. Here, we present an integrated multi-scale 3D imaging approach that traverses from millimeter-scale live-cell light microscopy to nanoscale volume electron microscopy. Performing the 3D cell culture in a single carrier amenable to all imaging steps provides a continuum resolution view of the same organoid. Our approach allows us to follow organoid growth, probe their morphology with fluorescent markers, identify cells of interest and analyze their ultrastructure. We demonstrate this workflow on mouse and human derived 3D cell cultures. The continuum resolution imaging pipeline is thus suited to foster both basic and translational organoid research by simultaneously exploiting the advantages of light and electron microscopy. 


\section{Introduction}

Cell culture systems are indispensable tools to understand diverse cellular functions in a wide range of fundamental and pre-clinical research. When modelling tissue-specific processes or multifaceted diseases like cancer, conventional two-dimensional (2D) cell cultures fail to fully recapitulate the tissue complexity and the cellular heterogeneity of the normal and cancerous tissue (Baker and Chen, 2012; Debnath and Brugge, 2005). Conversely, three-dimensional (3D) cell cultures can deliver more accurate representations of cell-cell and cell-extracellular matrix (ECM) interactions (Simian and Bissell, 2017), cell communication (Kretzschmar and Clevers, 2016) and cell division (Knouse et al., 2018). 3D culture cancer models allow studying tumour microenvironment, cell heterogeneity, and cell invasion (Kapałczyńska et al., 2016; Zanoni et al., 2020) as well as the effect of anti-cancer drugs (Lancaster and Huch, 2019). 3D cell cultures are also amenable to the vast majority of cell and molecular biology technologies used for 2D cultures. Thus, patient or cell line derived 3D culture systems have the potential to bridge the gap between reductionist $2 \mathrm{D}$ models and organismal models that are more expensive and inaccessible for many imaging or high-throughput methods. 3D cultures can be established by (i) anchorage-independent cell suspensions, (ii) scaffold-based approaches by embedding in natural or synthetic ECM, (iii) tissue slices, or (iv) air-liquid interfaces (Koledova, 2017; Shamir and Ewald, 2014). Commonly, 3D cultures are derived from immortalized cell lines, induced pluripotent stem cells (iPSs), embryonic stem cells (ESCs), or primary cells dissociated from animal or human tissues. The cells are seeded in a support matrix, typically consisting of a laminin-rich hydrogel termed Matrigel (Hughes et al., 2010). Cell lines cultured in 3D matrices form spheroids, while organoids are derived from stem cells or primary cells originating from healthy or diseased tissues (Fatehullah et al., 2016; Simian and Bissell, 2017).

Imaging is a powerful tool to study $3 \mathrm{D}$ cell culture complexity and to reveal processes that reflect intricate behaviour within whole organisms. Bright-field microscopy enables long-term imaging of organoid development at a micrometer scale with minimal phototoxicity (Hof et al., 2021). Confocal and light-sheet microscopy permit the study of specific cellular processes by using fluorescent reporters for cell types, organelles, or proteins (Day and Davidson, 2009), antibody labelling of antigens (Im et al., 2019), or cell-permeable dyes (Ettinger and Wittmann, 2014). However, due to the inherent opaque nature of organoids, optical clearing procedures are often necessary to render large multicellular samples transparent and thus amenable to light microscopy imaging (Tomer et al., 2014). Clearing procedures, on the other hand, require chemical fixation that degrades the fluorescent signal over-time (Ertürk et al., 2012), largely limiting live-cell imaging and post-fixation fluorescence-based imaging. Moreover, to ensure effective fluorescent labelling, organoids must be extracted from the Matrigel (Dekkers et al., 2019) thus preventing post clearing image correlation. Most importantly, diffraction-limited imaging modalities cannot visualize ultrastructural detail within cells. For this purpose, researchers generally rely on $2 \mathrm{D}$ imaging of ultrathin sections examined at high magnifications by transmission electron microscopy (TEM) (Knott and Genoud, 2013). Such data are often associated with low throughput, sectioning artifacts and limited field of view on the specimen. The biggest limitation of this technique, when imaging 3D cell cultures, is the lack of volumetric information that is essential to thoroughly appreciate multicellular organisation in space. Conversely, focused ion beam-scanning electron microscopy (FIB-SEM) is an ideal solution 
to this problem because of its marked different micromachining principle. This method involves progressive specimen slicing by FIB surface ablation, followed by SEM imaging (Giannuzzi and Stevie, 2005; Heymann et al., 2006). FIB-SEM thus generates a series of images that can cover a relatively wide field of view at nanometer-scale resolution, and extensive z-slicing yields volumes of 3D ultrastructural information (Heymann et al., 2006; Narayan and Subramaniam, 2015). However, none of the imaging methods alone can provide a comprehensive understanding of cells in the context of the complex multicellular environment of an organoid, prohibiting integration of structural and functional data across different spatial and temporal scales. While correlative approaches that combine the power of light and electron-based imaging modalities have been extensively applied for 2D cultures (Arnold et al., 2016; Kukulski et al., 2011), technical shortcomings limit their implementation in organoid research. The major challenge stems from the diverse requirements these imaging modes have, which are rarely met by one single specimen preparation.

Modern electron microscopy (EM) specimen preparation relies on cryogenic sample fixation, that uses low temperatures to stabilize the sample under fully hydrated physiological conditions. To obtain optimal preservation of fine ultrastructure, freezing must retain the water molecules in an amorphous solid state in a process called vitrification (Dubochet and McDowall, 1981). For objects up to 400 microns thick, including 3D cell cultures, vitrification is achievable by high-pressure freezing (HPF) (Moor, 1987). Here, samples that are sandwiched between two metal disks, commonly known as planchets or HPF carriers. The specimen is then pressurized to 2000 bar followed by cooling with liquid nitrogen within 200 milliseconds. Next, the cryo-fixed samples are subjected to freeze-substitution (Kellenberger, 1987 ) and infiltrated with embedding resin, which is then polymerized while the samples are stained with heavy metals to provide contrast in EM imaging (Biel et al., 2003). A milde variation of this procedure preserves endogenous fluorescent signal in the sample, while still providing sufficient contrast for FIB-SEM imaging (Ronchi et al., 2021). This enables precise targeting of specific regions of interest (ROI) in large sample volumes by fluorescence imaging after embedding. Such preparation methods are easily applicable for specimens that are amenable to manual handling for cryo-fixation, including small model organisms or dissected tissues. However, the fragility of $3 \mathrm{D}$ organoids grown in soft matrices precludes such manipulation.

It is therefore beneficial to perform the 3D cell cultures in sample carries that are directly suited for cryo-fixation. Here, we show that it is possible to culture 3D cell systems in HPF carriers, and develop a seamless workflow to image the cultures from their initial development to highresolution FIB-SEM. This establishes a multiscale 3D imaging pipeline in which the same organoid is tracked across the different modalities and scales. We applied the developed pipeline to mouse primary mammary gland organoids, human breast cancer spheroids, and patient-derived colorectal cancer organoids.

\section{Results}

\section{Establishing a multiscale imaging pipeline}

In multimodal imaging pipelines, EM has the most stringent requirements for specimen preparation due to its high resolving power. HPF is the only approach to preserve the native structure of multicellular samples. We employed common biocompatible gold-coated copper 
HPF carriers as a readily available support, and show that it is suitable for long-term 3D cell culture and all subsequent imaging modalities (Fig 1A). We used $200 \mu \mathrm{m}$ deep HPF carriers featuring a $0.6 \mathrm{~mm}^{3}$ sample volume and $3.14 \mathrm{~mm}^{2}$ surface area that is exposed to the culture medium. This results in a surface-to-volume ratio that is about 8 times larger than conventional $3 \mathrm{D}$ cell culture gels in tissue culture (TC) dishes, and was therefore not expected to hinder efficient diffusion of oxygen and nutrients to the organoids. We performed the 3D culture directly in HPF carriers by pipetting in the recess 1-2 $\mu \mathrm{l}$ of cell suspension mixed with Matrigel. We then placed the carriers in multi-well TC-dishes supplied with the culture medium. To aid handling and to prevent floating of the small carriers, we fixed the carriers with a droplet of Matrigel to $18 \mathrm{~mm}$ glass coverslips. With this setup, we could directly monitor the 3D cell culture growth by stereo-microscopy up to a period of 24 days and correlate it with subsequent live-cell confocal imaging. Next, we performed high-pressure freezing at chosen time points of culture. Freeze-substitution, Lowicryl HM20 resin embedding and heavy metal staining that retained fluorescence signal in the specimen were carried out in preparation for room temperature FIB-SEM (Ronchi et al., 2021). The resin block was then imaged by confocal fluorescence microscopy to relocate features previously identified in the specimen by live-cell imaging and define ROls. We generated landmarks on the resin block surface by two-photon laser to facilitate identification of the ROIs in the subsequent FIB-SEM, and guide their highresolution imaging (Ronchi et al., 2021). We then acquired FIB-SEM data of the targeted areas, resulting in an integrated imaging pipeline covering all spatial scales from $10^{-3}$ to $10^{-9}$ $\mathrm{m}$. Depending on the amount of image data required, the pipeline may require up to 10 days, with the freeze-substitution being the longest step for sample preparation (Fig 1B).

The transfer of very delicate samples, such as organoids, into HPF carriers is a cumbersome procedure which requires organoids removal from the ECM (TRIFFO et al., 2008). This may alter their morphology and prevents image registration pre and post cryo-fixation. By establishing 3D cultures directly in HPF carriers, 3D cell culture, sample handling and all imaging steps require minimal manual intervention.

\section{HPF carriers are compatible with diverse 3D cell culture models}

To validate the compatibility of the developed pipeline for organoid research, we examined three diverse types of $3 \mathrm{D}$ cell cultures. These included models of primary healthy and tumorigenic epithelia derived from mouse, patient derived human colorectal cancer, and a tumour cell line. We established a culture of primary mouse mammary organoids (Havas et al., 2017; Jechlinger et al., 2009) from single-cell suspensions derived from transgenic mice carrying H2B-mCherry as a nuclear fluorescent marker (Fig 2A). Within 14 days, the cells formed luminal sphere-shaped cystic structures in both HPF carriers and TC dishes (Fig 2A, $B)$. To assist imaging by different light microscopes, we marked with blunt-tip tweezers the bottom of the HPF carriers prior to Matrigel deposition (Fig 2A). The fluorescent signal of the transgenic H2B-mCherry reporter allowed us to inspect the organoids distribution and morphology (Fig 2C). Primary mammary organoids grown in the HPF carriers displayed the expected single layer of epithelial cells arranged around a lumen, comparable with standard culture conditions (Fig 2C) (Alladin et al., 2020). Immunostaining of tissue-specific differentiation markers on fixed samples directly in the HPF carriers showed that the organoids are predominantly composed of luminal mammary epithelial cells expressing Keratin-8 (K8) and a few basal/myoepithelial mammary epithelial cells expressing Keratin-14 (K14) (Fig 2 D). In addition, we performed immunofluorescent staining for the apical-basal cell polarity marker epithelial Cadherin (E-CAD) and the tight-junction protein Zonula occludens-1 (ZO-1) which 
localized in the apical cell membrane on organoids cultured in HPF carriers and TC dishes. These showed that the lumen is lined by the apical membrane of the polarized epithelial cells, while the basal side faces ECM in both HPF carriers (Fig. 2E) and TC dishes cultures (Fig. $2 F$ ). These data confirm that characteristics of polarized epithelia are preserved in HPF carriers. Moreover, activation of oncogene expression by doxycycline addition in the HPF carriers was reminiscent of the tumour-induced organoids grown on the dishes (Alladin et al., 2020) (Fig. S1).

Patient-derived colorectal cancer organoids grow within a week as irregularly-shaped clusters (Fig 2G). These organoids too display similar morphology when grown in HPF carriers and in standard 3D gels on TC dishes (Fig 2G,H). Fluorescence live-cell confocal imaging of patientderived organoids in HPF carriers stained with nuclear dye Hoechst-33342 showed multiple cell layers with small lumina (Fig 2l). The organoids expressed E-CAD and the Cytokeratin-20 (CK20) marker specific for colon tissue (Kummar et al., 2002) (Fig 2J). Immunofluorescent staining in both HPF carriers (Fig 2K) and TC dishes (Fig 2L) for E-CAD and ZO-1 showed that the expected apical-basal cell polarity is recapitulated in both culture conditions.

Human breast cancer cells BT-474 are a cell line which develops as regularly-shaped spheroids when embedded in Matrigel and cultured in HPF carriers for up to 14 days (Fig 2M). Confocal live-cell imaging confirmed that these spheroids form as a solid sphere without a lumen in HPF carriers (Fig $2 \mathrm{~N}$ ) and do not display apical-basal cell polarity when probed by immunofluorescent staining of E-CAD and ZO-1 (Fig 2O) (Florian et al., 2019).

These results demonstrate that primary mouse mammary gland organoids, patient-derived colorectal cancer organoids and human breast cancer spheroids grown in HPF carriers display morphologies, cellular composition, and cell polarity similar to those grown on TC dishes. Therefore, HPF carriers are applicable for a range of 3D cell cultures, and are compatible with confocal microscopy performed on live and fixed organoid samples as the first step in a multiscale imaging pipeline.

\section{Small molecule live dyes for correlative imaging of 3D cell cultures}

Genetically accessible cell lines or animal models are frequently used to generate 3D cell cultures, allowing the introduction of fusion proteins as fluorescent reporters. Genetic tagging is however difficult to obtain in human-derived organoids. In figure 2, we demonstrated the broad applicability of organoid cell culture in HPF carriers on organoids derived from transgenic mice with or without fluorescent reporters, and with two widely studied humanderived systems. In organoids devoid of transgenic fluorescent reporters, we employed cellpermeable fluorescent dyes (hereafter live dyes) to visualize specific cellular structures by fluorescence confocal microscopy. Small-molecule live dyes are often incompatible with many fixation procedures. Specifically in EM preparations, they are washed off by organic solvents during chemical fixation and dehydration, unless they are supplied during the embedding step (Biel et al., 2003), which in turn prevents the correlation with pre-embedding live-cell imaging. However, mass spectrometry studies showed that live dyes may be resilient to the freeze substitution (Pfeiffer et al., 2000). Thus, we tested four live dyes with different chemical and physical properties: Hoechsts-33342, SiR-actin, FM4-64 and BODIPY 493/503. We used a combination of Hoechst-33342 to mark cell nuclei and one of the 3 different dyes. All live dyes infiltrated organoids grown in HPF carriers, allowing live imaging of cell nuclei, of SiR-actin stained cortex in human BT474 spheroids (Fig 3, Fig S2A-C) and in mammary gland organoids 
(Fig S2D-F), of BODIPY 493/503 stained lipid droplets in mammary gland tumor organoids (Fig S2G-I), and of cell membranes stained with FM4-64 in patient-derived colorectal cancer organoids (Fig S2J-L). Furthermore, the live dyes were largely preserved in the gentle freezesubstitution procedure used here: in agreement with their chemical properties, hydrophilic dyes were not affected by freeze substitution (Hoechsts-33342, SiR-actin, Fig 3B,C) allowing detection of fluorescence before and after freeze substitution. The fluorescence signal of the membrane marker FM4-64 appeared diffuse after freeze-substitution, but permitted discerning cell boundaries (Fig 3D,E). The hydrophobic lipid droplet marker BODIPY 493/503 was removed during the washing step with organic solvents in freeze substitution (Fig 3F). Conversely, HPF alone (Fig 3G) did not affect the fluorescence signal of lipophilic live dyes, as determined by direct imaging of the HPF samples with a cryo-confocal microscope (Fig $3 \mathrm{H}, \mathrm{I})$. Thus, live dyes present an alternative for genetic tagging in fluorescence-based correlative imaging in $3 \mathrm{D}$ cultures.

\section{FIB-SEM volume imaging of 3D organoids}

Following localization of ROls in the resin block by confocal imaging and generation of surface landmarks by two-photon laser branding in the same microscope, we performed FIB-SEM volume imaging on selected organoids. It is well known that effective vitrification by HPF varies from sample to sample. The embedding ECM or medium, sample size, and even the metabolic state of different cells within the same specimen affect the quality of vitrification (Sitte et al., 1987). Ice crystals growth can induce organelles collapse, segregation of compartments and aggregation of macromolecules. Our EM data showed that organoids display a different freezing behaviour from their embedding medium, the Matrigel mix, which reveals fiber-like features (Fig 4A, Movie 1). Fully grown primary mouse mammary organoids developed in 6-8 days in culture, during which cells polarized and self-arranged in mono-layered acinus. Before reaching this stage, cells are intertwined in several layers within crowded acini (lumen up 15$20 \%$ of the whole organoid volume) (Fig 4A, Movie 1). FIB-SEM data of organoid at this stage of growth generally showed good ultrastructural preservation with fine details of centrioles, mitochondria and condensed chromatin (Fig 4B). Conversely, in monolayer organoids, the lumen comprises the majority (up to $60 \%$ ) of the organoid volume (Fig $2 \mathrm{C}$, Fig S2E, Fig 4C). The polarized cells displayed typical freezing damage in nuclei, and commonly in the cytosol, with prominent freeze substitution artifacts at cell-cell and cell-ECM interfaces (Fig 4C,D).

Despite the inherent heterogeneity of freezing quality expected in HPF, the preservation of the cellular ultrastructure seemed to improve with increased local density of cells within the organoids and reduced size of the lumen. To provide a semi-quantitative description of the freezing quality, we prepared thin sections from the HPF 3D cultures for TEM imaging allowing larger scale imaging. We prepared thin sections from HPF carriers with 3D cultures of doxycycline-induced tumorigenic (Mouse Ind), and healthy (Mouse $\mathrm{NI}$ ) primary mouse mammary organoids, human spheroids (BT474) and patient-derived colorectal cancer organoids (Fig 4E,F). After freeze-substitution and resin embedding (see material and methods section for details) we cut sections orthogonally with respect to the HPF carrier surface aiming for organoids located in the bulk of Matrigel (Fig S3A). We performed TEM imaging of 6-10 organoids per sample type. This allowed us to score the quality of structural preservation in relation to the organoid morphology, by exploiting the marked differences of organoid architecture and cell packing (Fig 4.E,F). We estimated the cellular packing as the ratio between the organoids total cytosol area divided by the number of cells, approximated by counting the nuclei in the TEM sections. Our thin sectioning TEM survey shows that more 
compact organoids (human colorectal cancer and breast cancer spheroids) show better ultrastructural preservation with a minimum of $60 \%$, a median of $83 \%$ for the colorectal organoids and $75 \%$ for breast cancer spheroids (Fig $4 . E, F)$. Conversely, we obtained lower success in high-pressure freezing primary mouse mammary organoids with large lumina, varying between $23 \%$ and $17 \%$ of median ultrastructural preservation (Fig $4 \mathrm{E}, \mathrm{F}$ ). This is consistent with reports of shrinkage/collapse of mono-layered or cystic organoids with large lumina during optical clearing procedures for light microscopy applications (Dekkers et al., 2019).

Human colorectal cancer organoids grow as large and compact structures. They protrude into the Matrigel with multiple branches featuring one lumen (Fig S3). Unlike the mouse organoids, colorectal organoids show better preserved cell ultrastructure and a much larger number of cells with little or no freezing damage (Fig S3). The human breast cancer spheroid represented the densest system without a lumen and exhibited minimal freezing damage (Fig S4). Damageh was constrained to the center of the sample, as expected from theoretical considerations of heat transfer during HPF. Structural damage was confined to cell nuclei, and occasional cytosolic segregation was restricted to one or two cell layers at the spheroid center (Fig S4). Overall, our data suggest that more compact organoids suffer less freeze damage despite the use of an identical embedding matrix and carrier dimensions (Fig 4.F).

Achieving optimal structural preservation during HPF is generally optimized for each specimen type (Möbius et al., 2010). This typically consists of embedding or infiltrating the sample with release agents like 1-hexadecene or lecithin, and cryo-protectants, such as glycerol, dimethyl sulfoxide, bovine serum albumin, yeast paste, fish gelatin, polyvinylpyrrolidone, Dextran, sucrose, or Ficoll. These compounds work by directly inhibiting the nucleation of ice crystals or increasing the sample cooling rates by suppressing the heat released in the crystallization process (McDonald et al., 2007). We therefore sought to improve the vitrification of the least preserved 3D culture sample encountered, namely luminal mouse mammary organoids, by introducing cryo-protectants prior to cryo-fixation. We found that simply dipping the HPF carriers for 1 minute before HPF in Cellbanker 2 (a common freezing medium, see material and methods section for details) or in $20 \%$ Ficoll $(70.000 \mathrm{MW}$ ) dissolved in cell culture medium, greatly improved the sample quality after HPF (Fig S5).

In summary, Matrigel-embedded organoids are challenging samples for successful highpressure freezing because of the high water content within the embedding medium. On the other hand, Matrigel provides the necessary physiological environment to perform 3D cell cultures. We found that organoids with higher cellular packing are more resilient against freeze damage and provide adequate ultrastructural preservation. Moreover, we show that different organoid systems require optimisation that can be achieved by careful selection of additives prior to high-pressure freezing.

\section{Multimodal imaging of 3D organoids with continuum spatial resolution}

Using the HPF carriers as a container for 3D cell culture allowed minimal manual intervention during their development and facilitated seamless correlation of all the described modalities to achieve continuum resolution imaging from millimeter to nanometer scale. By performing the 3D cell culture of human breast cancer spheroids directly in HPF carriers, we monitored organoid growth by stereo microscopy (Fig 5A). Each carrier was stained using live dyes, tracked and transferred into $35 \mathrm{~mm}$ glass-bottom dishes for the acquisition of live-cell confocal 
volumes (Fig 5B,C). We inspected the overall architecture of 3D cell culture and selected a specific organoid for higher resolution imaging. Subsequently, the samples were highpressure frozen and processed with a gentle freeze-substitution protocol to preserve the fluorescence signal (Fig 5D-F). The specific organoids or cells of interest were brought closer to the resin block surface by iterative confocal imaging and subsequent trimming of the excess of resin by ultramicrotomy (Ronchi et al., 2021). Two-photon laser surface branding of the resin surface aided the targeting for FIB-SEM data acquisition with micrometer precision (Fig $5 \mathrm{E})$. We identified the surface branded targets in the SEM, performed metal deposition and trench preparation following established protocols (Fig 5 G-J). FIB-SEM data was acquired with a sampling of $8 \times 8 \mathrm{~nm} /$ pixel at the image plane, and $10 \mathrm{~nm}$ FIB slicing representing sampling along the third dimension (Fig $5 \mathrm{~K}-\mathrm{L}$, Movie 2). The correlation across all imaging steps enabled overlay of the fluorescence with the FIB-SEM data (Fig $5 \mathrm{~K}-\mathrm{M}$ ).

\section{Discussion}

We describe an integrated multiscale imaging pipeline across optical and electron microscopes that enables easy and seamless correlative investigations in the emerging 3D cell culture model systems. Performing 3D cell culture directly in HPF carriers presented several advantages. First, it avoids manual transfer of the delicate samples from standard TC dishes to specialised carriers for cryo-fixation. This guarantees that the sample remains unperturbed from the beginning of cell seeding until high-pressure freezing. Second, performing 3D cell culture directly in HPF carriers avoids the need of dedicated carriers (Hötte et al., 2019) and additional embedding or mounting for confocal and light-sheet imaging (Huang et al., 2021). Therefore, the use of HPF carriers enabled us to correlate the growth of the 3D cell culture (stereo-microscopy), the identification of cellular structures of interest (livecell fluorescence confocal imaging), with the subsequent post-embedding imaging and twophoton laser branding, to ultimately guide FIB-SEM volume imaging.

Because the water phase transition at supercooling rates is not yet completely understood, one has to optimize the freezing conditions for each sample type. ECM-embedded 3D cell cultures, especially cystic organoids with large lumina, are among the most difficult samples to vitrify. We found that supplementing the culture medium with ready-to-use Cellbanker 2 or $20 \%$ Ficoll greatly improved the quality of the cryo-fixation for mouse primary luminal mammary gland organoids that showed the most serious freezing artifact. These protocols could serve as useful reference for ultrastructural studies on cystic or monolayered organoids.

HPF carriers provide a high surface area/volume ratio, which allows infiltration of the live 3D cultures with diverse small molecules (100 to 1000 Da molecular weight). We could infiltrate small molecules like the drug doxycycline (Fig S1) and commonly used live dyes (Fig S2). We further establish that a number of live dyes are resilient to freeze-substitution. Rochi et al., (Ronchi et al., 2021) recently demonstrated that it is possible to exploit the fluorescent signal of transgenic fusion proteins to target FIB-SEM acquisitions. However, the production of transgenic fluorescently-tagged reporters can be impractical, such in the case of patientderived samples. Therefore, small molecule live dyes can be used to identify specific phenotypes (Day and Davidson, 2009) paving the way to study culture systems that are not easily accessible to genetic engineering. Therefore, 3D cell cultures performed in HPF carriers 
are amenable to drug screening and treatment, with the advantage of being directly accessible to imaging by both light and electron microscopy. Because of the unspecific and broadspectrum binding of the uranyl-acetate stain, FIB-SEM can visualise the vast majority of subcellular components at nanomenter resolution. Future efforts for integrating such imaging approaches into high-throughput platforms being developed for organoid research will facilitate expanding organoid phenotyping to the ultrastructural level and aid in deriving mechanistic understanding of tissue-specific processes or drug effects. With these developments, we envision that patient-derived tumour organoids will become amenable to ultrastructural analysis to study multicellular interactions during drug treatment. This has the potential to achieve a deeper understanding of drug resistance, e.g. via loss of polarity/epithelial-mesenchymal transition (Croix and Kerbel, 1997; Dongre and Weinberg, 2019; van Staalduinen et al., 2018) and therapies modulating cell-cell contacts (Vucetic et al., 2020). Co-cultures of patient-derived colorectal carcinoma organoids have also been used to interrogate the effects of anaerobic microbiota on tumor progression (Pleguezuelos-Manzano et al., 2020). The use of fluorescently marked patient-derived organoids together with differentially marked associated taxa allows now the ultrastructural investigation on detrimental tissue/bacteria interactions (Schmidt et al., 2018) as well as the detrimental loss of epithelial barrier integrity (Yu, 2018).

In summary, our integrated imagining pipeline achieves a resolution continuum from the millimeter to nanometer scale, and is applicable to commonly used 3D cell cultures. While sample-specific optimization will be required to achieve optimal ultrastructural preservation for different organoid types, the developed pipeline and protocols could be easily adopted by EM labs that routinely practice HPF and FIB-SEM volume imaging, broadening their capability to the study of 3D cultures. We envision that the continuum resolution pipeline further establishes the basis for applications in full cryogenic regime with the aim to expand the resolution range to the molecular scale.

\section{Acknowledgments}

FIB-SEM volumes presented in the movies and in figures 4 and 5 will be made available in the Electron Microscopy Public Image Archive (EMPIAR). We acknowledge support from the EMBL electron microscopy core facility, Advanced Light Microscopy facility, IT Services and the EMBL Laboratory for Animal Resources. We thank Davide Floris and Sara Goetz for discussions about image processing, Martina Dees for kindly providing Hoechst-33342, Mark Schneider and Christa Stolp from Thoraxklinik University Hospital Heidelberg for providing tissue samples for deriving human colorectal cancer organoids. The prototype cryo-confocal fluorescence light microscope was developed in collaboration with Leica Microsystems. ED was supported by a fellowship from the EMBL Interdisciplinary (EI4POD) programme under Marie Skłodowska-Curie Actions COFUND (847543). SG was supported by a fellowship from the EMBL Interdisciplinary (EI3POD) programme under Marie Skłodowska-Curie Actions COFUND (664726). YS, MJ, and JM acknowledge support from the EMBL. JM acknowledges funding from the European Research Council (3DCellPhase- 760067).

\section{Ethics}

The use of animals in this study was approved by the Institutional Animal Care and Use Committee (approval \# MJ160070). Animals were treated at the EMBL in agreement with National and International laws and policies. All effort were made to minimize animal numbers 
in accordance with Russell and Burch's (1959) principle of (3Rs) reduction and highest ethical standards. Patient biosamples were provided by the Lung Biobank Heidelberg, member of the biomaterial bank Heidelberg (BMBH) in accordance with the regulations of the $\mathrm{BMBH}$ and the approval of the ethics committee of the University of Heidelberg (study S-270/2001 - biobank vote).

\section{Authors contributions}

EDI: Conceptualization, methodology, Validation, Formal analysis, Investigation, Data curation, Writing - original draft, Visualization, Project administration. MGM: Methodology, Validation, Investigation, Data curation, Project administration. SG: Methodology, Validation, Formal analysis, Investigation, Data curation, Visualization, Project administration. IZ: Validation, Investigation, Data curation. PR: Validation, Investigation, Data curation. YS: Supervision, Funding Acquisition, Project administration. MJ: Conceptualization, Supervision, Funding Acquisition, Project administration. JM: Conceptualization, Supervision, Funding Acquisition, Project administration. All the authors contributed to reviewing and editing the manuscript.

\section{Competing interests}

All authors declare no conflict of interest. 


\section{Figures}
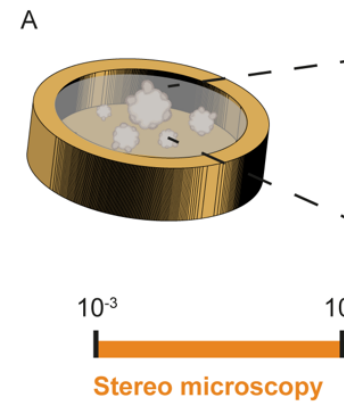
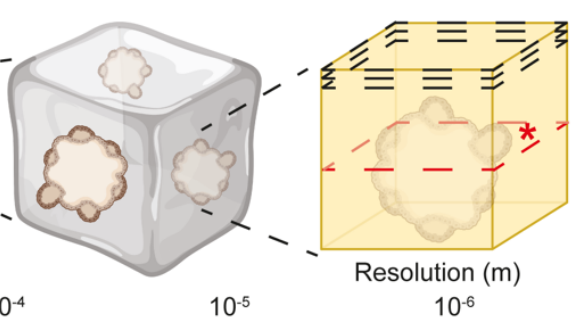

$10^{-6}$

Confocal microscopy
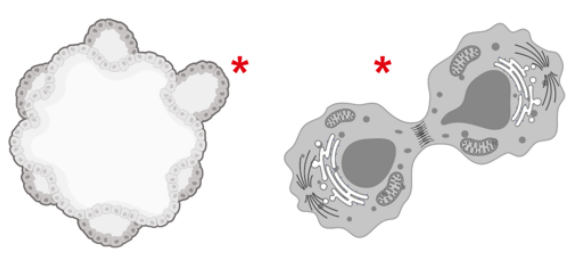

$10^{-7}$

$10^{-8}$

FIB-SEM volume imaging

B

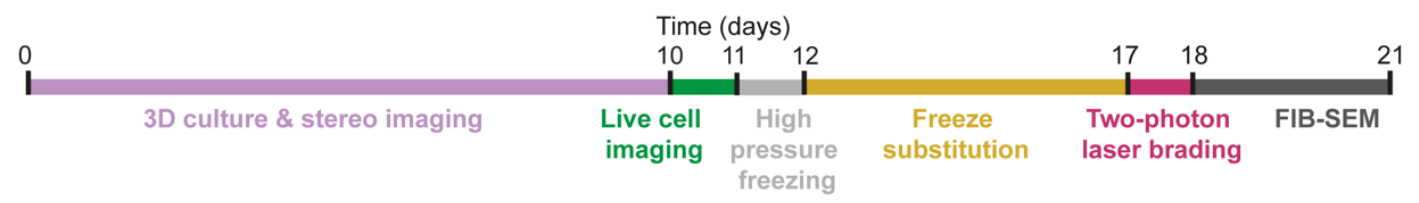

Figure 1. 3D cell cultures in HPF carriers enable continuous correlative imaging from the millimeter to the nanometer scale. A, a multiscale imaging pipeline of 3D cell cultures in HPF carriers encompasses millimeter-scale stereo light microscopy, 3D confocal fluorescence microscopy prior to and following cryo-fixation, and nanometer-scale FIB-SEM volume imaging. Asterisks indicate single cells that can be targeted and followed throughout the imaging pipeline. B, timeline of 3D cell culture together with sample preparation and imaging regime. 
Primary mouse luminal mammary gland organoids

A HPF carrier Stereo microscopy
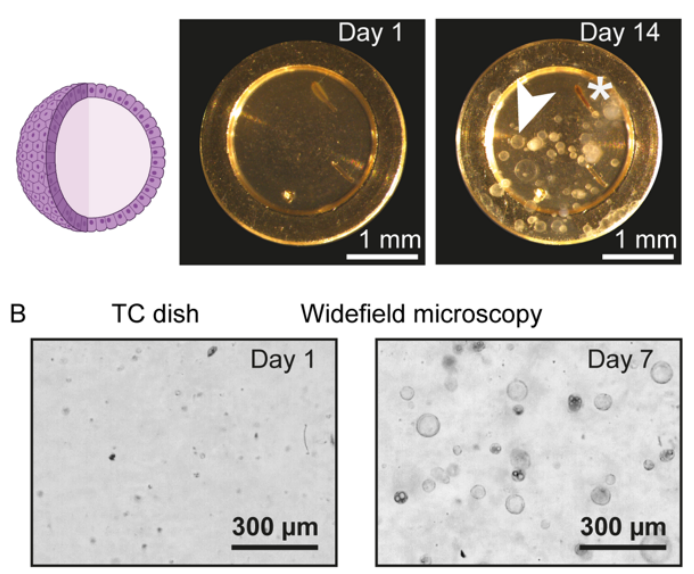

Patient-derived colorectal cancer organoids

G HPF carrier Stereo microscopy
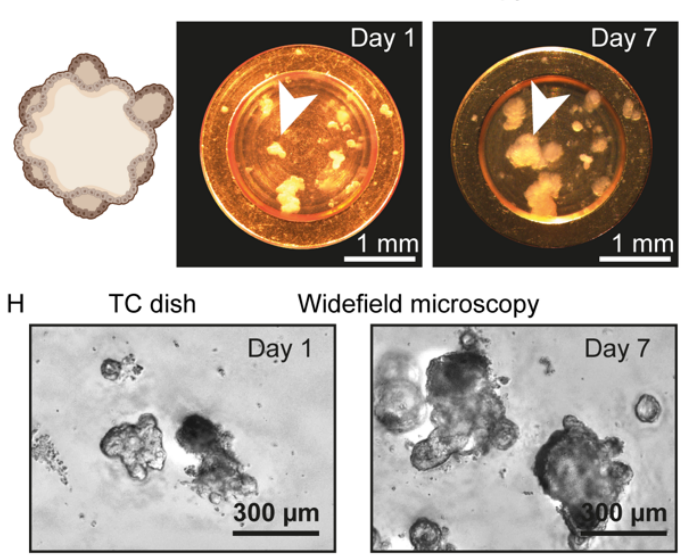

Human breast cancer BT-474 spheroids

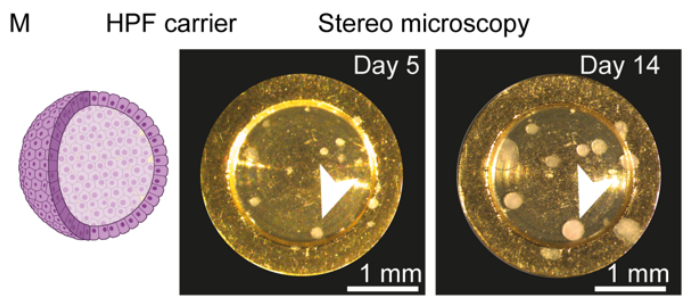

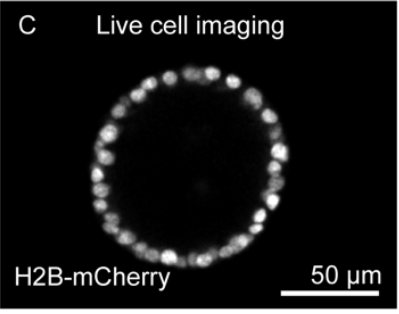
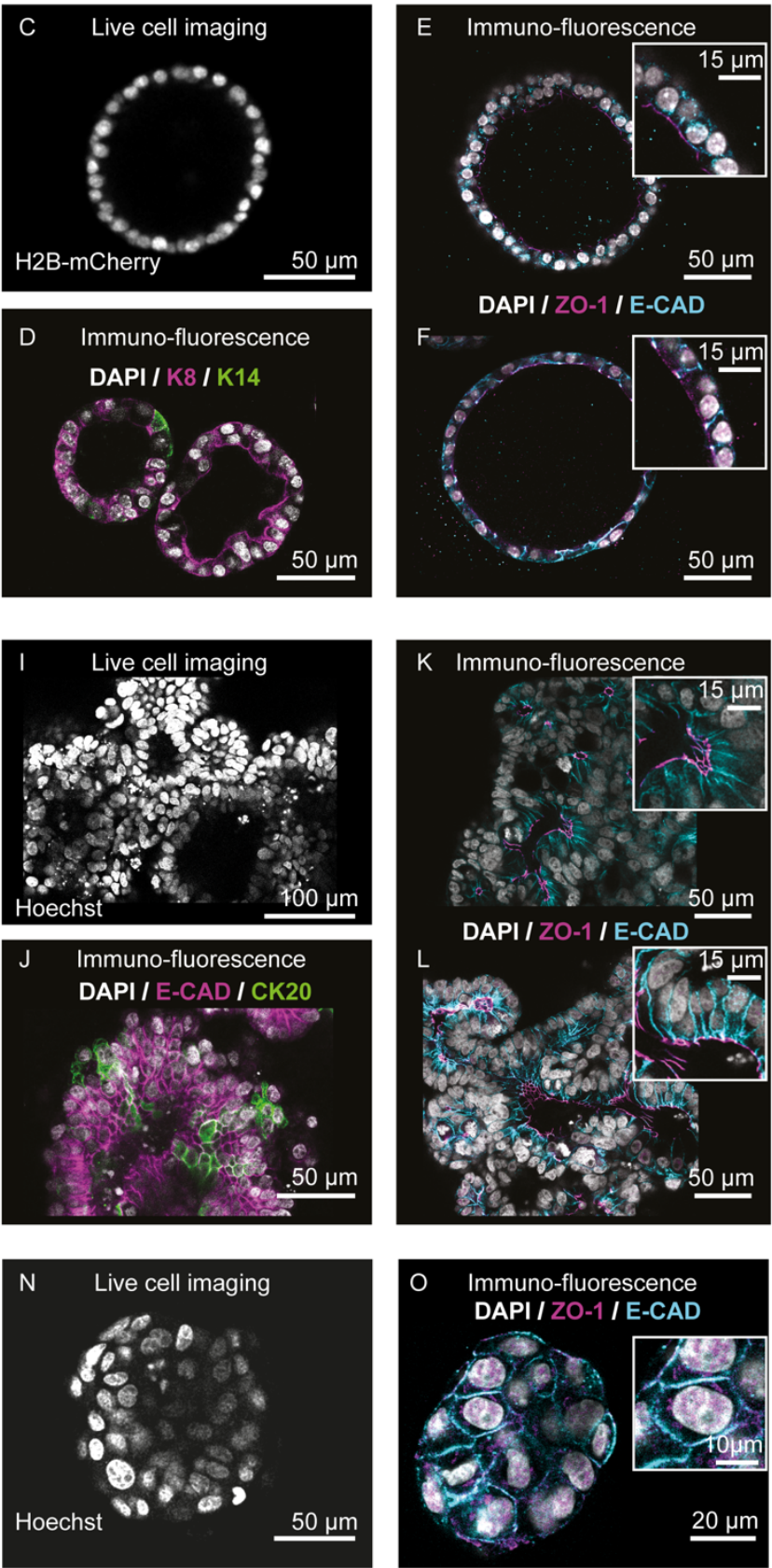

Figure 2. Preservation of 3D multicellular morphologies in HPF carriers. A-F, Comparison of primary mouse mammary organoids growth in HPF carriers (A) and standard tissue culture (TC) dishes (B) at the indicated time points of the culture. Asterisks and arrowheads, respectively, indicate marks of the HPF carriers and a single organoids. C, a confocal plane of an organoid from (A) expressing mCherry-tagged histone H2B. D, immunostaining in HPF carriers with tissue-specific markers: luminal Keratin-8 (K8) and basal/myoepithelial Keratin-14 (K14); cell nuclei stained with DAPI. E, F, immunostaining of tissue-specific markers: the cell-adhesion protein epithelial cadherin (E-CAD, cyan), tight junctions (ZO-1), and cell nuclei (DAPI) of organoids grown in HPF carriers (E) and in TCdishes (F). Insets: details of cell-cell interfaces. $\mathbf{G}, \mathbf{H}$, comparison of patient-derived colorectal cancer organoids growth at the indicated time points of the culture in HPF carriers and TC dishes, respectively. Arrowheads indicate growth of a single organoid. I, a confocal plane of an organoid from (G) with cell nuclei labelled with Hoechst-33342. J, immunostaining in HPF 
carriers with tissue-specific markers: E-cadherin (E-CAD) and Cytokeratin-20 (CK20), a colon cell marker; cell nuclei stained with DAPI (white). K, L, immunostaining of tissue-specific polarity markers E-cadherin (E-CAD, cyan), tight junctions (ZO-1, magenta), and cell nuclei (DAPI, white) of patient-derived organoids grown in HPF carriers (K) and in TC-dishes (L). Insets: details of cell-cell interfaces. $\mathbf{M}$, human breast cancer spheroids growth in HPF carriers at the indicated time points. Arrowheads indicate growth of a single organoid. $\mathbf{N}$, a confocal plane of a spheroid from (M) with nuclei marked with Hoechst-33342. O, immunostaining of tissue-specific markers: E-cadherin (E-CAD, cyan), tight junctions (ZO-1, magenta), and cell nuclei (DAPI, white) of spheroids grown in HPF carriers. Insets: details of cell-cell interfaces. 

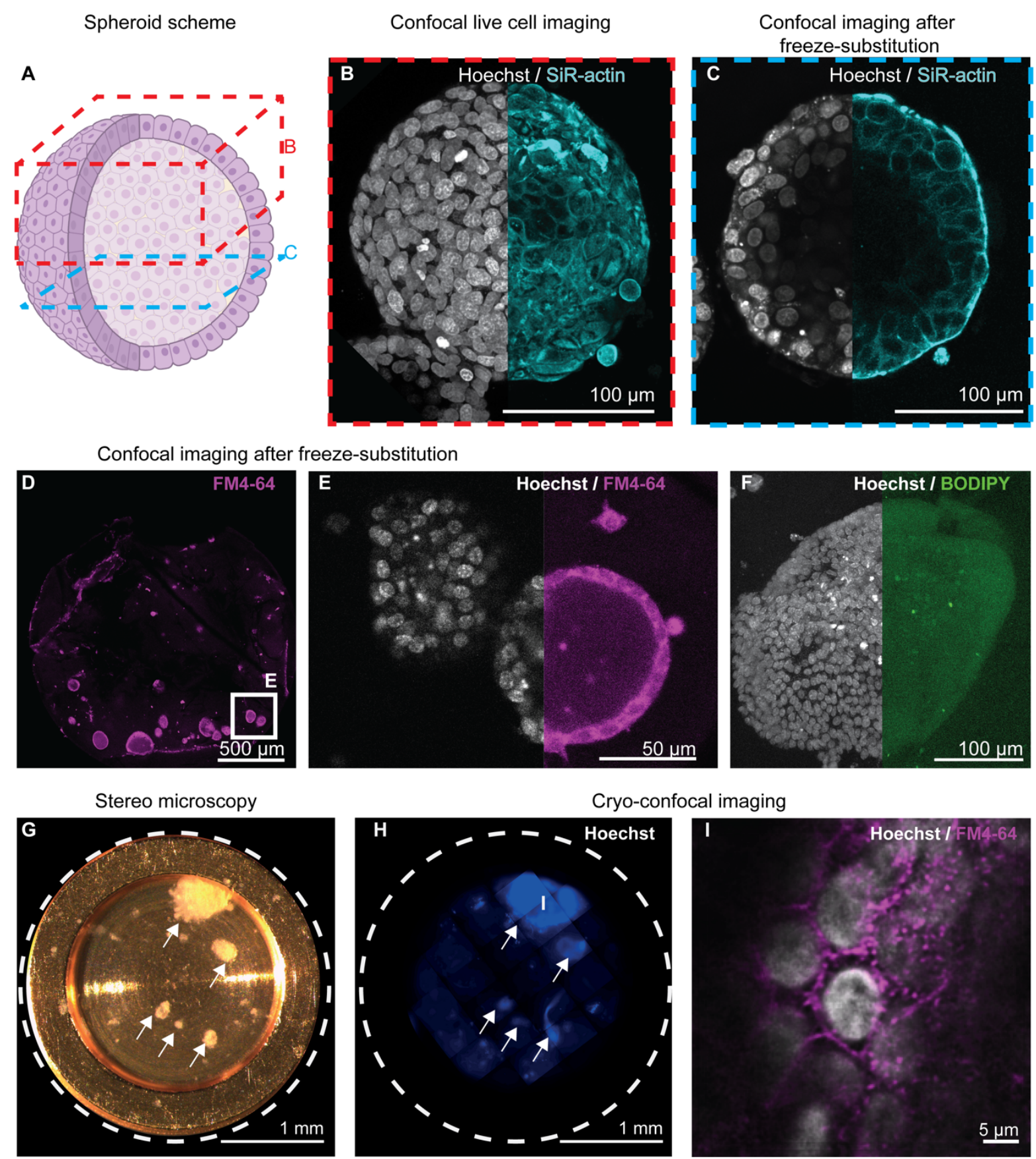

Figure 3. Preservation of fluorescent live dyes in cryo-fixation and freeze-substitution. A, schematic representation of a BT474 human breast cancer spheroid. Locations of confocal imaging data before (red, B) and after (blue, C) freezing and freeze-substitution are indicated. B, maximum intensity projection of the live spheroid confocal volume stained with live dyes for detection of cell nuclei (Hoechst-33342, grayscale) and F-actin (SiR-actin, cyan). C, a confocal plane of the exposed resin block surface. D-F, primary mouse mammary organoids after freeze-substitution with membrane and nuclear live dyes (FM4-64 in magenta, Hoechsts33342 in grayscale, respectively) in confocal plane (D), and single organoids (E). F, Tumorinduced mouse mammary organoid with nuclear (Hoechst-33342, greyscale) and lipid-droplet (BODIPY 497/503, green) live dyes. G-I, light microscopy at cryogenic temperature of patientderived organoids (white arrows) cultured in HPF carriers before $(\mathbf{G})$ and after $(\mathbf{H})$ high- 
bioRxiv preprint doi: https://doi.org/10.1101/2021.07.02.450855; this version posted July 3, 2021. The copyright holder for this preprint (which was not certified by peer review) is the author/funder, who has granted bioRxiv a license to display the preprint in perpetuity. It is made available under aCC-BY-NC-ND 4.0 International license.

pressure freezing. I, a cryo-confocal image of an organoid in $\mathbf{( H )}$ with nuclear (Hoechst-33342, grayscale) and membrane (FM4-64, magenta) live dyes. 

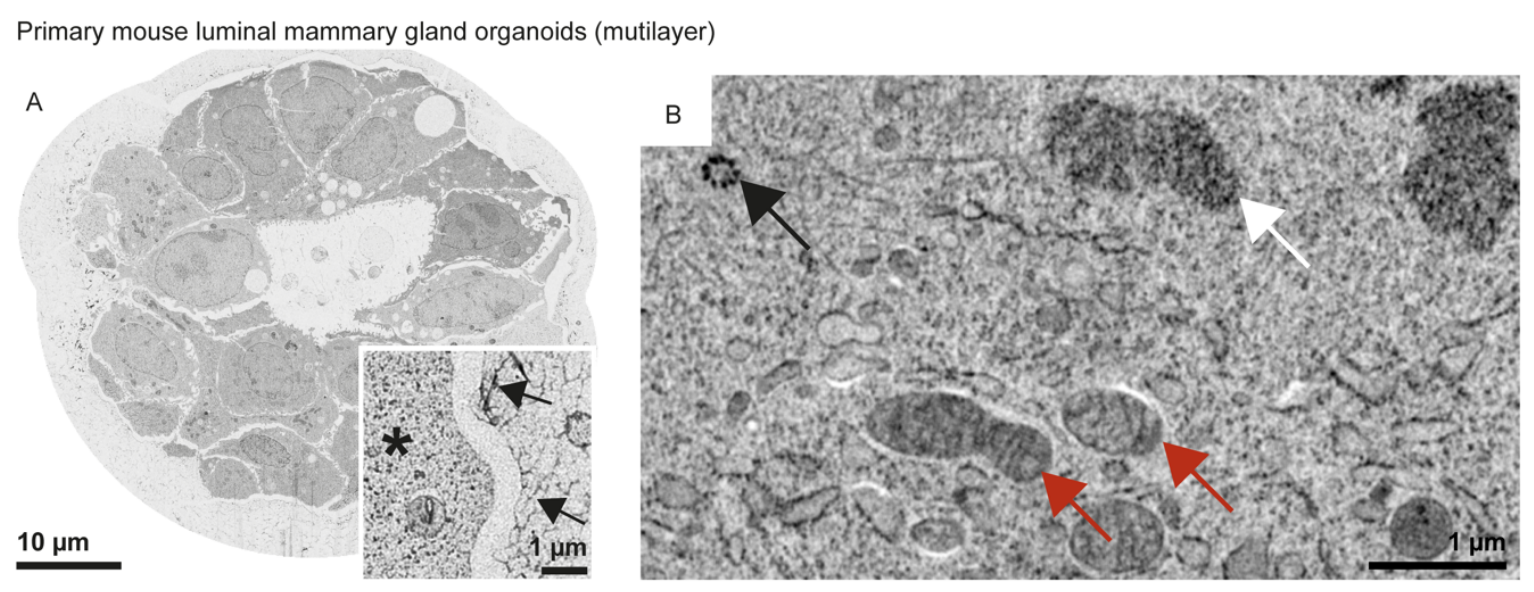

Primary mouse luminal mammary gland organoids (monolayer)
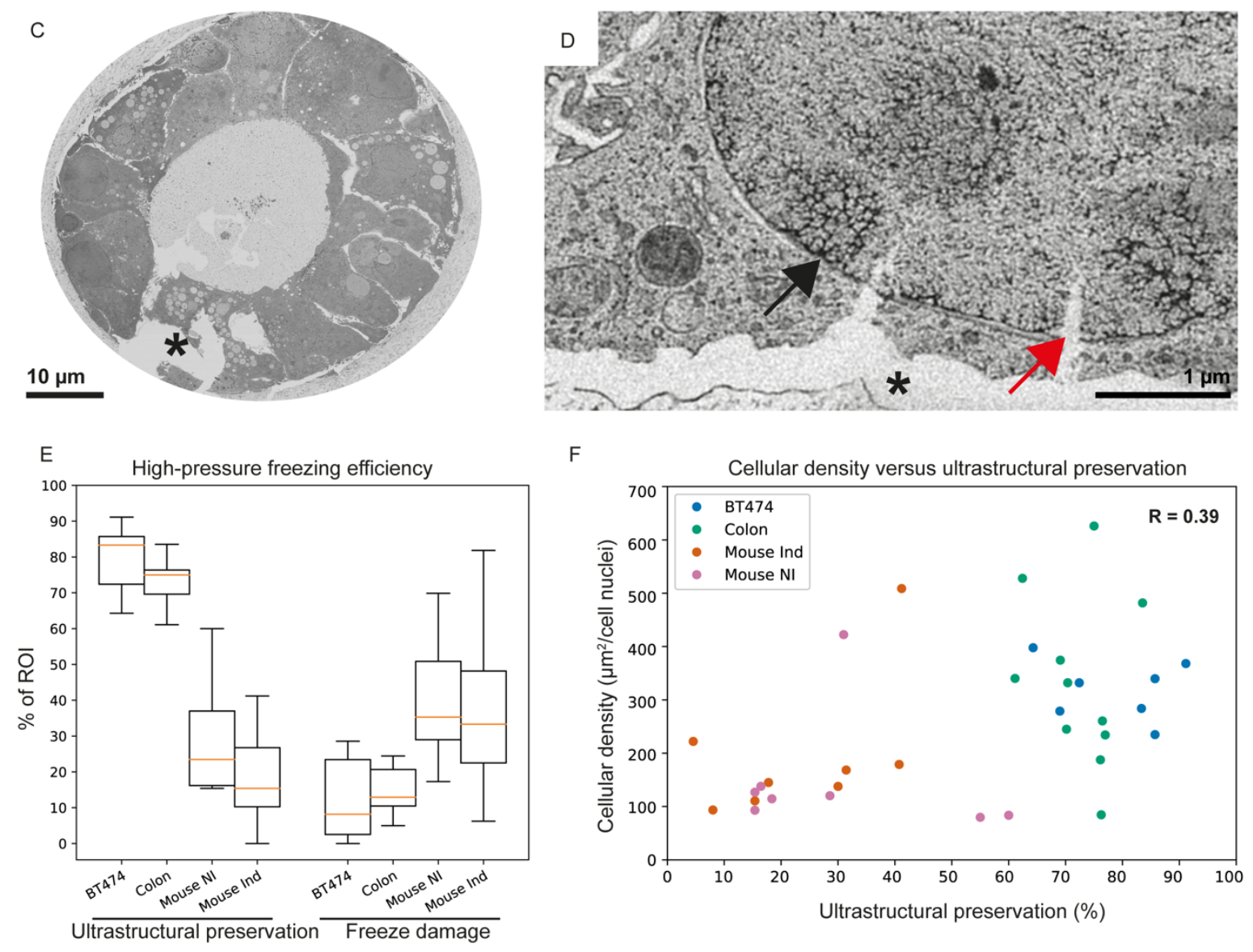

Figure 4. FIB-SEM of 3D cell cultures grown in HPF carriers. Examples of FIB-SEM slices of primary mouse mammary organoids. Multilayer organoids after seven days of culture (A) with details (inset) of Matrigel (arrowheads) versus cytoplasm (black asterisk) characteristic freezing. A well-preserved cell (B) with condensed chromatin (white arrow), a centriole (black arrow), and mitochondria (red arrows). Monolayer organoids (C, D) show freezing damage (black arrow) and extensive cracks (red arrow), with membrane detachment (black asterisks). E, Quantification of HPF efficiency by thin sections TEM and its correlation with cell density within organoid (F) for the four 3D culture types embedded in Matrigel: human breast spheroids (BT474), human colorectal cancer organoids (Colon), doxycycline-induced tumorigenic (Mouse Ind), and healthy (Mouse $\mathrm{NI}$ ) primary mouse mammary gland organoids. 


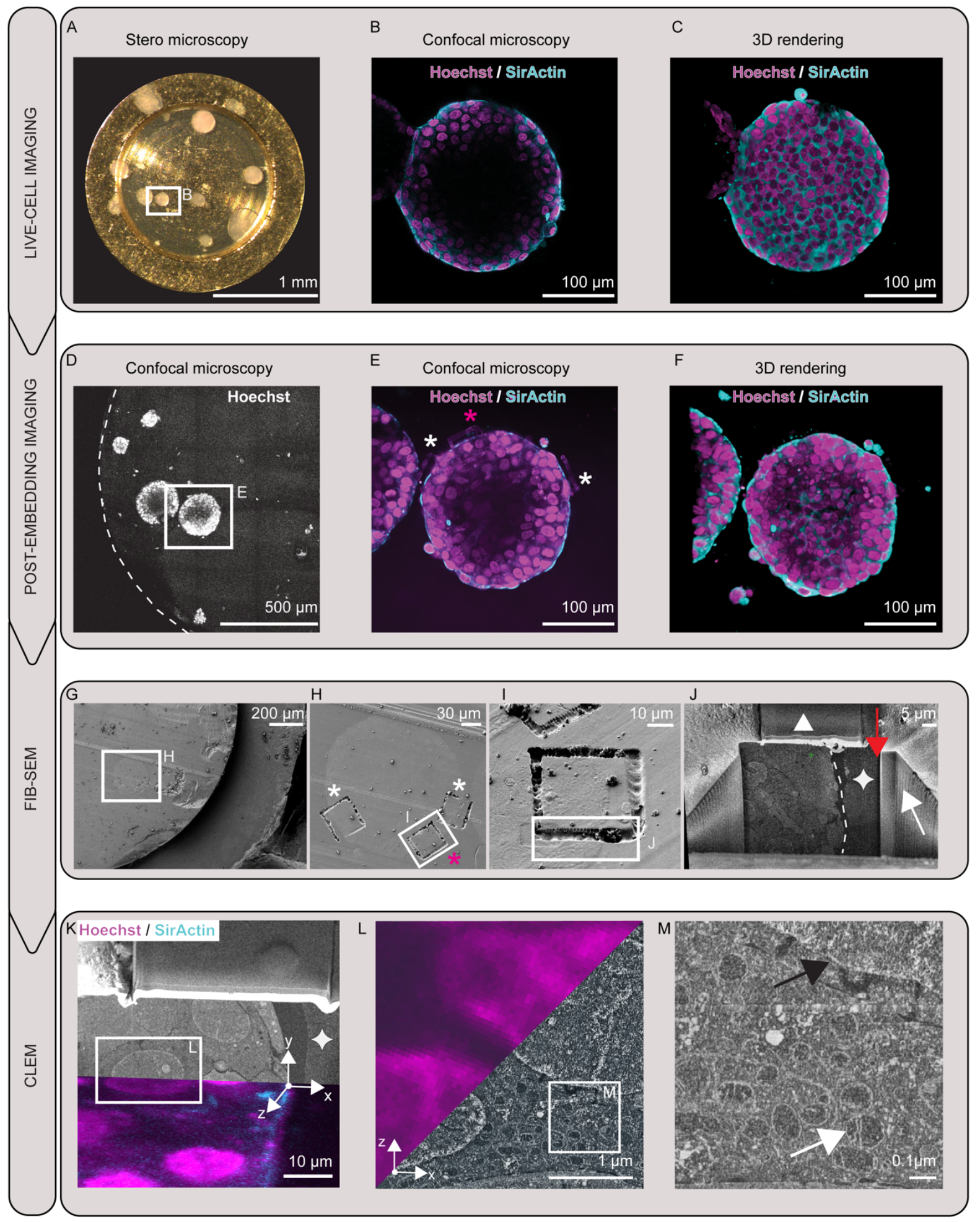

Figure 5. Correlative imaging of 3D cell culture from the millimeter to the nanometer scale. A, stereomicroscopy of BT474 human breast cancer spheroid cultured in HPF carrier. Confocal slice $(\mathbf{B})$ and $3 \mathrm{D}$ rendering $(\mathbf{C})$ of the indicated spheroid in A. Cell nuclei (Hoechst33342, magenta) and F-actin (SiR-actin, cyan). D. Maximum intensity projection tiled scan of the specimen from $\mathbf{A}$ after freeze-substitution. Frame indicates the spheroid imaged in $\mathbf{B}, \mathbf{C}$, and enlarged in E, F. E, Confocal plane after freeze substitution. Surface laser brandings were introduced at positions of interest (white asterisks) to guide FIB-SEM volume imaging (magenta asterisk). Cell nuclei (Hoechst-33342, magenta) and F-actin (SiR-actin, cyan). F, 3D 
rendering of $\mathbf{E}$. $\mathbf{G}$, SEM view of the organoid in $\mathbf{E}$; brandings $(\mathbf{H}$, white asterisks) and FIB-SEM target (magenta asterisk) before $(\mathbf{I})$ and after trench milling $(\mathbf{J})$ with details of the organoid outer edge (white dash) next to the embedding Matrigel (white star). The white triangle indicates the protective platinum layer above the milling volume. Red and white arrows point to the milling direction and the milling progression, respectively. $\mathbf{K}$, Correlated light and electron volume imaging of targeted cells from the organoid in $\mathbf{J}$ (magenta asterisk in $\mathbf{E}$ and H) next to embedding Matrigel (white star). $x, y$ directions are relative to the SEM imaging plane, orthogonal to the volume $(\mathrm{z})$ highlighted by the correlated fluorescence postembedding. L, Enlarged view (direction parallel to $\mathbf{y}$ in $\mathbf{K}$ ) of two nuclei from $\mathbf{K}$ with wellpreserved ultrastructural details (M) of a nucleus (black arrow) and mitochondria (white arrow). SEM images are displayed in negative contrast to aid comparison with fluorescent signal. 


\section{Materials and Methods}

\section{Animal experimentation}

Animals are treated at the European Molecular Laboratory in agreement with National and International laws and policies. All efforts are made to use the minimal number of animals as possible in accordance with Russell and Burch's (1959) principle of (3Rs) reduction and highest ethical standards. The IACUC (Institutional Animal Care and Use Committee) approved the work with these mice (approval \# MJ160070).

\section{Animals}

TetO-MYC/ MMTV-rtTA (D'Cruz et al., 2001) and TetO-Neu/ MMTV-rtTA (Moody et al., 2002) mouse strains were bred to obtain TetO-MYC/ TetO-Neu/ MMTV-rtTA animals. A reporter R26-H2B-mCherry mouse strain line (Abe et al., 2011) (RIKEN, CDB0239K) was crossed in to establish the experimental line TetO-MYC/ TetO-Neu/ MMTV-rtTA/ R26-H2B-mCherry in FVB background. The housing of all animals used in this study was performed in the Laboratory Animal Resources facility at EMBL Heidelberg, according to the guidelines and standards of the Federation of European Laboratory Animal Science Association (FELASA).

\section{D Cultures}

Every sample type described hereafter shares, in both HPF carries and standard TC dishes, culture conditions as well as sample manipulation, only differing in the volume of the Matrigel and the carrier on which they are seeded.

\section{Primary mouse mammary organoids}

Mammary glands were dissected from 7-9 weeks old female virgin mice and collected in a 15 $\mathrm{ml}$ falcon. For dissociation of the tissue, the mammary glands were digested overnight at 37 ${ }^{\circ} \mathrm{C}$ and $5 \% \mathrm{CO}_{2}$ in a loosely capped $50 \mathrm{ml}$ falcon with $5 \mathrm{ml}$ of DMEM/F12 (Lonza) supplemented with $25 \mathrm{mM}$ HEPES, 1\% Penicillin Streptomycin solution (ThermoFisher), 750 units of Collagenase Type III (Worthington Biochemical Corporation) and $20 \mu \mathrm{g}$ of Liberase TM (Roche). After 16 hours digestion, cells were washed with DMEM/F12 (Lonza) supplemented with 25 mM HEPES, 1\% Penicillin Streptomycin solution (ThermoFisher). Pellet was then trypsinized with $5 \mathrm{ml}$ of $0.25 \%$ Trypsin-EDTA (ThermoFisher) to obtain single cells following the published protocol (Jechlinger et al., 2009). To generate 3D cultures, we prepared in ice a master mix of Matrigel Growth Factors Reduced (Corning, 356231), Rat Collagen I (RnDSystems, 3447-020-01), and PBS following 4:1:1 proportion. We combined the cell suspension and matrix mix to obtain a concentration of 30,000 cells/100 $\mu$ l of master mix. We seeded a volume of $4 \mu \mathrm{l}$ in the $200 \mu \mathrm{m}$ deep HPF carriers, previously sterilised with $70 \%$ Ethanol and fixed with Matrigel (Corning), to an $18 \mathrm{~mm}$ glass coverslip. Then, each carrier was placed into a well of a 24-well plate (Corning) to allow the gels to polymerize at $37^{\circ} \mathrm{C}$ with $5 \% \mathrm{CO}_{2}$. After polymerization, we supplied gels with $1 \mathrm{ml}$ of Mammary Epithelial Cell Growth Medium (Promocell, c-21010) supplemented with Mammary Epithelial Cell Growth supplement (Sciencell, 7652 ) and incubated at $37^{\circ} \mathrm{C}$ in a humidified atmosphere with $5 \% \mathrm{CO}_{2}$. For induction of oncogene expression in the organoids, Doxycycline Hyclate (Sigma, D9891) was diluted into the growth medium at $600 \mathrm{ng} / \mathrm{ml}$ after 4 days of 3D cell culture. Growth media were exchanged every other day in both conditions. 


\section{Human colorectal cancer organoids}

Biosamples were provided by Lungbiobank Heidelberg member of the biomaterial bank Heidelberg $(\mathrm{BMBH})$ in accordance with the regulations of the $\mathrm{BMBH}$ and the approval of the ethics committee of the University of Heidelberg (study S-270/2001 - biobank vote). Metastatic lesion from a patient diagnosed with metastatic colorectal cancer was surgically removed and dissected from lung tissue. A tumor fragment of a minimum of $100 \mathrm{~mm}^{2}$ was used for the establishment of primary organoid culture. Briefly, tissue was mechanically dissociated using scalpel followed by pipetting through a $10 \mathrm{ml}$ pipette in a basal medium Advanced DMEM/F12 (Gibco), Primocin $50 \mu \mathrm{g} / \mathrm{ml}$ (Invivogen), 1\% GlutaMAX (Gibco), 1\% HEPES (Gibco), 1\% Penicillin Streptomycin solution (Gibco), N-Acetylcysteine $1.25 \mathrm{mM}$ (Sigma) supplemented with 1\% B27 supplement (Gibco). Next, the cell suspension was enzymatically digested for 2 hours at $37{ }^{\circ} \mathrm{C}$ in basal medium supplemented with Liberase $\mathrm{DH}$ (final concentration 0.28 Wünsch units $/ \mathrm{ml}$ ). The cell suspension was then filtered through $100 \mu \mathrm{m}$ and $40 \mu \mathrm{m}$ cell strainer. Single cells were seeded in Growth-Factor Reduced Matrigel (Corning) mixed with PBS (4:1) adjusted to a concentration of $17,000 \mathrm{cells} / \mu \mathrm{l}$ and cultured in culture medium (Advanced DMEM/F12 (Gibco) Primocin 50 $\mathrm{gg} / \mathrm{ml}$ (Invivogen), 1\% GlutaMAX (Gibco), 1\% HEPES (Gibco), penicillin $100 \mathrm{U} / \mathrm{ml}$ and streptomycin $100 \mu \mathrm{g} / \mathrm{ml}$ (Gibco), N-Acetylcysteine $1.25 \mathrm{mM}$ (Sigma) supplemented with 1\% B27 supplement (Gibco), $50 \mathrm{ng} / \mathrm{ml}$ Epidermal Growth Factor (EGF) (Peprotech), $100 \mathrm{ng} / \mathrm{ml}$ Noggin (Peprotech) and $500 \mathrm{nM}$ A83-01 (Tocris Bioscience). Organoids were passaged with Gentle Cell Dissociation Reagent (StemCell Technologies) according to manufacturer instructions. For imaging experiments, organoids from passage 4-10 were seeded on the HPF carriers using $2 \mu$ of organoid suspension in Matrigel/PBS (6:1).

\section{BT474 cell spheroids}

BT474 human cell line was obtained from American Type Culture Collection. Cells were grown in DMEM 1x $4.5 \mathrm{~g} / \mathrm{L}$ D-glucose FluoroBrite (Gibco) medium supplemented with 10\% inactivated FBS (Gibco), 1\% HEPES $1 \mathrm{M}$ (Gibco), 1\% Sodium Pyruvate $100 \mathrm{mM}$ (Gibco), 1\% MEM NEAA 100X (Gibco), 1\% L-Glutamine 200mM (Gibco), 1\% Penicillin Streptomycin solution (Gibco) and passaged using 0,05\% Trypsin-EDTA (Gibco). For spheroid formation and imaging experiments, cells were seeded on the HPF carriers in Growth-Factor Reduced Matrigel (Corning) mixed with PBS (6:1) at 500 cells/ $\mu$ l.

\section{Immunofluorescent staining}

Organoid cell culture was performed in HPF carriers (Wohlwend $\mathrm{GmbH}$ ) which were then transferred to a deactivated clear glass vial (Waters, 186000989DV), and fixed with 4\% PFA for 5 minutes followed by three washes of PBS. To prevent nonspecific antibody binding, the HPF carriers were incubated with $10 \%$ goat serum for 2 hours at room temperature. Primary antibody incubation was performed overnight at $4{ }^{\circ} \mathrm{C}$. Afterward, HPF carriers were washed with PBS three times for 10 minutes, and incubated with secondary antibodies and DAPI (Thermo Fisher, 62248, 1:1000). Samples were mounted using Prolong Gold with DAPI (Thermo Fisher, P36931). The following antibodies were used in this study: c-MYC (Cell Signaling Technologies, 5605, 1:800), ZO-1 (Thermo Fisher, 61-7300, 1:400), E-cadherin (Thermo Fisher, 13-1700, 1:200), Alpha6-integrin (Millipore, MAB1378, 1:100), Cytokeratin 8 (Troma-I, DSHB, 1:100), Cytokeratin 14 (Invitrogen, MA5-11599, 1:200) Cytokeratin 20 (Abcam, ab76126, 1:200), Alexa 488 (Invitrogen, A11034, 1:800), Alexa 568 (Invitrogen, A11031, 1:800), Alexa 647 (Invitrogen, 21247, 1:800). Mounts were imaged in HPF carriers on a Leica SP5 confocal microscope using a $63 \times 1.2$ NA water immersion lens and the LAS 
AF imaging software. HPF carriers were mounted facing the objective on top of a Nunc LabTek II chambered 1.5 borosilicate cover glass.

\section{Stereomicroscopy and widefield transmission imaging}

The 3D cell cultures in HPF carriers were maintained in 24-well plates and imaged in a Leica M125 C Stereomicroscope in dark field mode to enhance the HPF carriers contrast against the background. 3D cell culture grown in gels in TC-dishes over the time-course of the experiment were imaged using the widefield high-throughput Olympus ScanR microscope in transmission mode. Each well of the 24-well plate was imaged using 9 ROls per well, with 21 Z-stacks (100 $\mu \mathrm{m}$ of scanning step in Z). Images were acquired with 4x UplanSApo 0.16 NA Air objective in an environmental chamber at standard cell culture conditions $\left(37^{\circ} \mathrm{C}, 5 \% \mathrm{CO}_{2}\right)$. Projections of z-stacks and image stitching were done using Fiji software (Schneider et al., 2012).

\section{Live-cell confocal imaging}

For live cell imaging, HPF carriers were washed with PBS once, transferred to a $35 \mathrm{~mm}$ or 10 $\mathrm{mm}$ diameter cell culture dish (Greiner Bio One International, catalog number 627860, Kremsmünster, Austria). For samples devoid of genetic fluorescent tags, the HPF carriers were incubated for 20 minutes with the desired live dyes diluted in the growth medium to the following final concentrations: SiR-actin $100 \mu \mathrm{M}$ (Spyrochrome AG, catalog number SC001, Stein am Rhein-Switzerland), Hoechst-33342 $10 \mu \mathrm{M}$ (Thermo Fisher Scientific, Waltham, MA USA, catalog number H1399), FM4-64 $2 \mu \mathrm{M}$ (Thermo Fisher Scientific, Waltham, MA USA, catalog number T13320) and BODIPY 493/503 $1 \mu \mathrm{M}$ (Thermo Fisher Scientific, Waltham, MA, catalog number D3922). The culture dish was immersed in a drop of deionized water. HPF carriers were flipped with the recess facing the microscope objective lens. Live cell imaging was performed at $37{ }^{\circ} \mathrm{C}$ and $5 \% \mathrm{CO}_{2}$ with a Zeiss LSM 780 NLO (Jena, Germany) equipped with a LD LCI Plan-Apochromat 25x 0.8 NA water immersion objective. To image the whole HPF carrier volume and locate single organoids, we first detected cell nuclei marked with Hoechst-33342 and acquired z-stacks with $6 \times 6$ tiles of $512^{2}$ pixels with $10 \%$ overlap, and 10 $\mu \mathrm{m}$ z-step. The final montage was stitched using ZEN-black software. For single organoids, we acquired z-stacks of $1024^{2}$ pixels at different z-steps ranging from 1.0 or $1.8 \mu \mathrm{m}$.

\section{High-pressure freezing, freeze-substitution, and two-photon laser branding}

We followed the procedure described in (Ronchi et al., 2021). Briefly, we high pressure froze 3D cell cultures in HPF carriers with a high-pressure freezer HPM 010 (AbraFluid). Before high-pressure freezing mouse organoids with cryo-protecting chemicals, the HPF carriers containing the 3D cell cultures were dipped for one minute either directly in Cellbanker 2 cryopreserving medium (catalog number 11891, AMSBIO, Cambridge - US) or in $20 \%$ Ficoll $70.000 \mathrm{MW}$ (catalog number F2878, Sigma, Merck KGaA, Darmstadt, Germany) diluted in Mammary Epithelial Cell Growth Medium (Promocell, c-21010). Next we performed freezesubstitution with $0.1 \%$ uranyl acetate (UAc) in acetone. After $72 \mathrm{~h}$ incubation at $-90{ }^{\circ} \mathrm{C}$, the temperature was increased to allow the reaction of uranyl-acetate with the biological material. We then rinsed the samples with pure acetone before infiltration of the resin lowicryl HM20 (Polysciences). Finally, we polymerized the resin with UV at $-25{ }^{\circ} \mathrm{C}$. The resin-embedded samples were then transferred in 35 or $10 \mathrm{~mm}$ cell culture dish (Greiner Bio One International 627860 , Kremsmünster, Austria) with water as immersion medium and imaged at an inverted Zeiss LSM 780 NLO microscope (Jena, Germany) equipped with a 25x Plan-Apochromat 25x 0.8 NA Imm Korr DIC multi immersion objective lens. Surface branding was performed with 
the 2-photon Coherent Chameleon Ultra II Laser (Santa Clara, USA) of the Zeiss LSM 780 NLO microscope and the "bleaching" function of ZEN black software.

\section{Cryo-Confocal microscopy}

Confocal stack acquisition under cryo-conditions was carried out with Leica TCS SP8 upright microscope (Leica microsystems CMS GmbH, Mannheim, Germany), controlled by Leica Application Suite $X$ 3.5.5.19976 software. The microscope was equipped with cryo-stage, insulated HC PL APO 50x 0.90 NA DRY objective, and Leica DFC365 FX camera. The highpressure frozen organoids in HPF carriers were inserted into the cartridge (Leica microsystems CMS GmbH, Mannheim, Germany) with sample side facing the objective. All sample loading and transfer operations were conducted under cryo-conditions, in liquid nitrogen vapor phase, using a dedicated loading/transfer unit (Leica microsystems CMS $\mathrm{GmbH}$, Mannheim, Germany). The loading and transfer steps were carried out in a humiditycontrolled room $(10 \%)$ to minimize ice contamination on the sample. After the sample was deposited on the microscope stage pre-cooled to $-195^{\circ} \mathrm{C}$, we determined the approximate $\mathrm{z}$ position of the sample surface in wide-field illumination mode. Using Matrix MAPS+CLEM2 application within the Matrix Screener module, we acquired 100 microns deep (steps of $2 \mu \mathrm{m}$ ) z-stack tiles (x/y with $20 \%$ overlapping) to produce a stitched 3D overview image of the whole carrier at the desired wavelengths (Leica Application Suite $X$ 3.5.5.19976). This helped to determine ROls for subsequent confocal stacks acquisition. Confocal stacks were acquired in two channels: $405 \mathrm{~nm}$ at 50\% power Diode laser (HyD1 detector $410 \mathrm{~nm}-504 \mathrm{~nm}$ ) and 552 $\mathrm{nm}$ at 50\% power OP SL laser (HyD 3 detector $660 \mathrm{~nm}-778 \mathrm{~nm}$ ). Pinhole $1 \mathrm{AU}$, zoom 1, pixel format 1056x1056, pixel size in $x y=0.22 \mu \mathrm{m}$, in $z=0.372 \mu \mathrm{m}$, bidirectional scan at $200 \mathrm{~Hz}$. Z-stack of $50 \mu \mathrm{m}$ depth were acquired with a z-step system optimized for the $405 \mathrm{~nm}$ wavelength. Confocal stacks were processed with the Lightning deconvolution software module (Leica Application Suite X 3.5.5.19976) at default settings and a number of iterations set to 5 for both channels.

\section{FIB-SEM}

After confocal imaging and branding for targeting, the blocks were mounted on a SEM stub using silver conductive epoxy resin (Ted Pella). After mounting, the blocks were gold sputtered with a Quorum Q150R S coater. FIB/SEM imaging was performed on a Zeiss CrossBeam XB540 or XB550. Briefly, a platinum coat was deposited over the area marked by the laser branding. Auto-tuning marks were milled on the platinum surface and highlighted with carbon. Large trenches were milled with $30 \mathrm{nA}$ FIB current and surface polished with $7 \mathrm{nA}$ or $15 \mathrm{nA}$ currents. Precise milling during the run was achieved with currents of either $700 \mathrm{pA}$ or $1.5 \mathrm{nA}$. For all experiments, the SEM imaging was done with an acceleration voltage of $1.5 \mathrm{kV}$ and a current of $700 \mathrm{pA}$, using a back-scattered electron detector. For single cells within organoids, data were acquired either at $8 \mathrm{~nm}$ isotropic or $8 \times 8 \times 10 \mathrm{~nm}(\mathrm{x}, \mathrm{y}, \mathrm{z})$ sampling. For the acquisition of entire organoids, voxels of $15 \times 15 \times 20 \mathrm{~nm}$ were found to be the best compromise between the achievable resolution/field-of-view ratio and milling stability.

\section{Thin sectioning TEM}

Blocks prepared as described above were sectioned with an ultramicrotome (Leica UC7) and $70 \mathrm{~nm}$ sections were collected on formvar coated slot grids. Organoid TEM images were acquired without post-staining using a Jeol 2100 Plus operated at $120 \mathrm{kV}$. The organoids were identified at 400X mag (32 nm pixel size). Subsequently, single organoids were imaged by fitting a montage of tiles at $2000 \mathrm{X}$ mag $(6.7 \mathrm{~nm}$ pixel size) using SerialEM (Schorb et al., 2019). 
The montages were stitched with the command justblend and, where necessary, manually corrected with Midas both implemented in Imod (Kremer et al., 1996).

\section{Data processing and rendering}

Unless otherwise specified, all microscopy data were processed with Fiji (Schneider et al., 2012). The contrast of electron microscopy data was enhanced using the Fiji plugin Enhance Local Contrast (CLAHE) using the following parameters: blocksize 63, 256 histogram bins and maximum slope of 3.0. When necessary, before contrast enhancement, FIB-SEM volumes were corrected for curtaining effect applying wavelet decomposition (Münch et al., 2009) implemented in the freely available software SerialFIB (Klumpe et al., 2021). Here we used coif3 type vertical wavelets with a decomposition sigma level of 8 and a sigma gaussian for vertical stripes dampening of 6 . In movie 3 , we show wavelet decomposition effectiveness using the particularly severe curtaining in the dataset shown in Video 1 in Ronchi et al., (Ronchi et al., 2021). Movies were made with Fiji and synchronized with the macro by Patrice Malscalchi (https://github.com/AiviaCommunity/ImageJ-Macros-Utilities). For light microscopy data 3D rendering, Imaris 9.6.0 with either "blend" or "shadow projection" rendering modes was used. All schematics were created with Biorender (Biorender.com) and Adobe illustrator.

\section{References}

Abe, T., Kiyonari, H., Shioi, G., Inoue, K.-I., Nakao, K., Aizawa, S., and Fujimori, T. (2011). Establishment of conditional reporter mouse lines at ROSA26 locus for live cell imaging. Genesis 49, 579-590.

Alladin, A., Chaible, L., Garcia del Valle, L., Sabine, R., Loeschinger, M., Wachsmuth, M., Hériché, J.-K., Tischer, C., and Jechlinger, M. (2020). Tracking cells in epithelial acini by light sheet microscopy reveals proximity effects in breast cancer initiation. Elife 9.

Arnold, J., Mahamid, J., Lucic, V., de Marco, A., Fernandez, J.-J., Laugks, T., Mayer, T., Hyman, A.A., Baumeister, W., and Plitzko, J.M. (2016). Site-Specific Cryo-focused lon Beam Sample Preparation Guided by 3D Correlative Microscopy. Biophys. J. 110, 860-869.

Baker, B.M., and Chen, C.S. (2012). Deconstructing the third dimension - how 3D culture microenvironments alter cellular cues. J. Cell Sci. 125.

Biel, S.S., Kawaschinski, K., Wittern, K.-P., Hintze, U., and Wepf, R. (2003). From tissue to cellular ultrastructure: closing the gap between micro- and nanostructural imaging. J. Microsc. 212, 91-99.

Croix, B. St., and Kerbel, R.S. (1997). Cell adhesion and drug resistance in cancer. Curr. Opin. Oncol. 9, 549-556.

D'Cruz, C.M., Gunther, E.J., Boxer, R.B., Hartman, J.L., Sintasath, L., Moody, S.E., Cox, J.D., Ha, S.I., Belka, G.K., Golant, A., et al. (2001). c-MYC induces mammary tumorigenesis by means of a preferred pathway involving spontaneous Kras2 mutations. Nat. Med. 7, 235239.

Day, R.N., and Davidson, M.W. (2009). The fluorescent protein palette: tools for cellular imaging. Chem. Soc. Rev. 38, 2887.

Debnath, J., and Brugge, J.S. (2005). Modelling glandular epithelial cancers in threedimensional cultures. Nat. Rev. Cancer 5, 675-688.

Dekkers, J.F., Alieva, M., Wellens, L.M., Ariese, H.C.R., Jamieson, P.R., Vonk, A.M., Amatngalim, G.D., Hu, H., Oost, K.C., Snippert, H.J.G., et al. (2019). High-resolution 3D 
imaging of fixed and cleared organoids. Nat. Protoc. 14, 1756-1771.

Dongre, A., and Weinberg, R.A. (2019). New insights into the mechanisms of epithelialmesenchymal transition and implications for cancer. Nat. Rev. Mol. Cell Biol. 20, 69-84.

Dubochet, J., and McDowall, A.W. (1981). VITRIFICATION OF PURE WATER FOR ELECTRON MICROSCOPY. J. Microsc. 124, 3-4.

Ertürk, A., Becker, K., Jährling, N., Mauch, C.P., Hojer, C.D., Egen, J.G., Hellal, F., Bradke, F., Sheng, M., and Dodt, H.-U. (2012). Three-dimensional imaging of solvent-cleared organs using 3DISCO. Nat. Protoc. 7, 1983-1995.

Ettinger, A., and Wittmann, T. (2014). Fluorescence live cell imaging. In Methods in Cell Biology, pp. 77-94.

Fatehullah, A., Tan, S.H., and Barker, N. (2016). Organoids as an in vitro model of human development and disease. Nat. Cell Biol. 18, 246-254.

Florian, S., Iwamoto, Y., Coughlin, M., Weissleder, R., and Mitchison, T.J. (2019). A human organoid system that self-organizes to recapitulate growth and differentiation of a benign mammary tumor. Proc. Natl. Acad. Sci. 116, 11444-11453.

Giannuzzi, L.A., and Stevie, F.A. (2005). Introduction to Focused lon Beams (Boston, MA: Springer US).

Havas, K.M., Milchevskaya, V., Radic, K., Alladin, A., Kafkia, E., Garcia, M., Stolte, J., Klaus, B., Rotmensz, N., Gibson, T.J., et al. (2017). Metabolic shifts in residual breast cancer drive tumor recurrence. J. Clin. Invest. 127, 2091-2105.

Heymann, J.A.W., Hayles, M., Gestmann, I., Giannuzzi, L.A., Lich, B., and Subramaniam, S. (2006). Site-specific 3D imaging of cells and tissues with a dual beam microscope. J. Struct. Biol. 155, 63-73.

Hof, L., Moreth, T., Koch, M., Liebisch, T., Kurtz, M., Tarnick, J., Lissek, S.M., Verstegen, M.M.A., van der Laan, L.J.W., Huch, M., et al. (2021). Long-term live imaging and multiscale analysis identify heterogeneity and core principles of epithelial organoid morphogenesis.

BMC Biol. 19, 37.

Hötte, K., Koch, M., Hof, L., Tuppi, M., Moreth, T., Verstegen, M.M.A., van der Laan, L.J.W., Stelzer, E.H.K., and Pampaloni, F. (2019). Ultra-thin fluorocarbon foils optimise multiscale imaging of three-dimensional native and optically cleared specimens. Sci. Rep. 9, 17292.

Huang, Q., Garrett, A., Bose, S., Blocker, S., Rios, A.C., Clevers, H., and Shen, X. (2021). The frontier of live tissue imaging across space and time. Cell Stem Cell 28, 603-622.

Hughes, C.S., Postovit, L.M., and Lajoie, G.A. (2010). Matrigel: A complex protein mixture required for optimal growth of cell culture. Proteomics 10, 1886-1890.

Im, K., Mareninov, S., Diaz, M.F.P., and Yong, W.H. (2019). An Introduction to Performing Immunofluorescence Staining. In Methods in Molecular Biology, pp. 299-311.

Jechlinger, M., Podsypanina, K., and Varmus, H. (2009). Regulation of transgenes in threedimensional cultures of primary mouse mammary cells demonstrates oncogene dependence and identifies cells that survive deinduction. Genes Dev. 23, 1677-1688.

Kapałczyńska, M., Kolenda, T., Przybyła, W., Zajączkowska, M., Teresiak, A., Filas, V., Ibbs, M., Bliźniak, R., Łuczewski, Ł., and Lamperska, K. (2016). 2D and 3D cell cultures - a comparison of different types of cancer cell cultures. Arch. Med. Sci.

Kellenberger, E. (1987). The Response of Biological Macromolecules and Supramolecular Structures to the Physics of Specimen Cryopreparation. In Cryotechniques in Biological Electron Microscopy, (Berlin, Heidelberg: Springer Berlin Heidelberg), pp. 35-63.

Klumpe, S., Fung, H.K.H., Goetz, S.K., Zagoriy, I., Hampoelz, B., Zhang, X., Erdmann, P.S., 
Baumbach, J., Müller, C.W., Beck, M., et al. (2021). A Modular Platform for Streamlining Automated Cryo-FIB Workflows. BioRxiv 2021.05.19.444745.

Knott, G., and Genoud, C. (2013). Is EM dead? J. Cell Sci. 126, 4545-4552.

Knouse, K.A., Lopez, K.E., Bachofner, M., and Amon, A. (2018). Chromosome Segregation Fidelity in Epithelia Requires Tissue Architecture. Cell 175, 200-211.e13.

Koledova, Z. (2017). 3D Cell Culture: An Introduction. In Methods in Molecular Biology, pp. $1-11$.

Kremer, J.R., Mastronarde, D.N., and McIntosh, J.R. (1996). Computer Visualization of Three-Dimensional Image Data Using IMOD. J. Struct. Biol. 116, 71-76.

Kretzschmar, K., and Clevers, H. (2016). Organoids: Modeling Development and the Stem Cell Niche in a Dish. Dev. Cell 38, 590-600.

Kukulski, W., Schorb, M., Welsch, S., Picco, A., Kaksonen, M., and Briggs, J.A.G. (2011). Correlated fluorescence and 3D electron microscopy with high sensitivity and spatial precision. J. Cell Biol. 192, 111-119.

Kummar, S., Fogarasi, M., Canova, A., Mota, A., and Ciesielski, T. (2002). Cytokeratin 7 and 20 staining for the diagnosis of lung and colorectal adenocarcinoma. Br. J. Cancer 86, 18841887.

Lancaster, M.A., and Huch, M. (2019). Disease modelling in human organoids. Dis. Model. Mech. 12.

McDonald, K.L., Morphew, M., Verkade, P., and Müller-Reichert, T. (2007). Recent Advances in High-Pressure Freezing. In Methods in Molecular Biology, pp. 143-173.

Möbius, W., Cooper, B., Kaufmann, W.A., Imig, C., Ruhwedel, T., Snaidero, N., Saab, A.S., and Varoqueaux, F. (2010). Electron Microscopy of the Mouse Central Nervous System. In Methods in Cell Biology, pp. 475-512.

Moody, S.E., Sarkisian, C.J., Hahn, K.T., Gunther, E.J., Pickup, S., Dugan, K.D., Innocent, N., Cardiff, R.D., Schnall, M.D., and Chodosh, L.A. (2002). Conditional activation of Neu in the mammary epithelium of transgenic mice results in reversible pulmonary metastasis. Cancer Cell 2, 451-461.

Moor, H. (1987). Theory and Practice of High Pressure Freezing. In Cryotechniques in Biological Electron Microscopy, (Berlin, Heidelberg: Springer Berlin Heidelberg), pp. 175191.

Münch, B., Trtik, P., Marone, F., and Stampanoni, M. (2009). Stripe and ring artifact removal with combined wavelet-Fourier filtering. Opt. Express 17, 8567.

Narayan, K., and Subramaniam, S. (2015). Focused ion beams in biology. Nat. Methods 12 , 1021-1031.

Pfeiffer, S., Vielhaber, G., Vietzke, J.-P., Wittern, K.-P., Hintze, U., and Wepf, R. (2000). High-Pressure Freezing Provides New Information on Human Epidermis: Simultaneous Protein Antigen and Lamellar Lipid Structure Preservation. Study on Human Epidermis by Cryoimmobilization. J. Invest. Dermatol. 114, 1030-1038.

Pleguezuelos-Manzano, C., Puschhof, J., Rosendahl Huber, A., van Hoeck, A., Wood, H.M., Nomburg, J., Gurjao, C., Manders, F., Dalmasso, G., Stege, P.B., et al. (2020). Mutational signature in colorectal cancer caused by genotoxic pks+ E. coli. Nature 580, 269-273.

Ronchi, P., Mizzon, G., Machado, P., D'Imprima, E., Best, B.T., Cassella, L., Schnorrenberg, S., Montero, M.G., Jechlinger, M., Ephrussi, A., et al. (2021). High-precision targeting workflow for volume electron microscopy. J. Cell Biol. 220.

Schmidt, T.S.B., Raes, J., and Bork, P. (2018). The Human Gut Microbiome: From 
Association to Modulation. Cell 172, 1198-1215.

Schneider, C.A., Rasband, W.S., and Eliceiri, K.W. (2012). NIH Image to ImageJ: 25 years of image analysis. Nat. Methods 9, 671-675.

Schorb, M., Haberbosch, I., Hagen, W.J.H., Schwab, Y., and Mastronarde, D.N. (2019).

Software tools for automated transmission electron microscopy. Nat. Methods 16, 471-477.

Shamir, E.R., and Ewald, A.J. (2014). Three-dimensional organotypic culture: experimental models of mammalian biology and disease. Nat. Rev. Mol. Cell Biol. 15, 647-664.

Simian, M., and Bissell, M.J. (2017). Organoids: A historical perspective of thinking in three dimensions. J. Cell Biol. 216, 31-40.

Sitte, H., Edelmann, L., and Neumann, K. (1987). Cryofixation Without Pretreatment at Ambient Pressure. In Cryotechniques in Biological Electron Microscopy, (Berlin, Heidelberg: Springer Berlin Heidelberg), pp. 87-113.

van Staalduinen, J., Baker, D., ten Dijke, P., and van Dam, H. (2018). Epithelialmesenchymal-transition-inducing transcription factors: new targets for tackling chemoresistance in cancer? Oncogene 37, 6195-6211.

Tomer, R., Ye, L., Hsueh, B., and Deisseroth, K. (2014). Advanced CLARITY for rapid and high-resolution imaging of intact tissues. Nat. Protoc. 9, 1682-1697.

TRIFFO, W.J., PALSDOTTIR, H., McDONALD, K.L., LEE, J.K., INMAN, J.L., BISSELL, M.J., RAPHAEL, R.M., and AUER, M. (2008). Controlled microaspiration for high-pressure freezing: a new method for ultrastructural preservation of fragile and sparse tissues for TEM and electron tomography. J. Microsc. 230, 278-287.

Vucetic, M., Daher, B., Cassim, S., Meira, W., and Pouyssegur, J. (2020). Together we stand, apart we fall: how cell-to-cell contact/interplay provides resistance to ferroptosis. Cell Death Dis. 11, 789.

Yu, L.C.-H. (2018). Microbiota dysbiosis and barrier dysfunction in inflammatory bowel disease and colorectal cancers: exploring a common ground hypothesis. J. Biomed. Sci. 25, 79.

Zanoni, M., Cortesi, M., Zamagni, A., Arienti, C., Pignatta, S., and Tesei, A. (2020). Modeling neoplastic disease with spheroids and organoids. J. Hematol. Oncol. 13, 97. 